\title{
COMPRENSIONES Y REFLEXIONES SOBRE LA ACCIÓN PSICOSOCIAL EN EL CONTEXTO EDUCATIVO
}

\author{
Jacqueline Vera Martinez y Red de Curso \\ Directora de Curso Acción Psicosocial en el Contexto Educativo \\ Programa de Psicología UNAD \\ Correo electrónico: jacqueline.vera@unad.edu.co \\ ORCID ID: https://orcid.org/0000-0002-7146-3227
}

Octubre de 2018

Palabras clave: Educación, acción psicosocial, legislación educativa, concientización, contexto educativo, institución educativa, papel del psicólogo,

El contexto escolar, una lectura compleja

Dentro de la experiencia a través de las diferentes generaciones, se encuentra la idea con aroma a certeza, de que la educación sólo es posible en las aulas de clase y que los protagonistas de este proceso son los docentes y estudiantes. Esta representación social se evidencia de diferentes maneras en los dibujos que representan la clase, en los juegos infantiles y en la expectativa cuando se va a iniciar un módulo, materia, curso o espacio académico nuevo.

Por esta razón, cuando se descubre que el contexto escolar no sólo se trata de un docente y sus estudiantes, sino, que da cuenta de las relaciones de los diferentes actores que incluyen además a los administrativos, los padres de familia y por supuesto a la comunidad en la que se 



Por lo anterior, el ejercicio del psicólogo en el contexto educativo desde la perspectiva de la acción psicosocial, busca promover el fortalecimiento de los miembros de la comunidad educativa y considerar la inclusión social, valorando a cada quien en su SER y en su individualidad, buscando bienestar en la comunidad, respeto de los derechos, responsabilidad, confiabilidad y sinergia para construir comunidades educativas cada vez más democráticas, que sustentan su proyección en la equidad, la diversidad, la justicia social y la capacidad de elegir, decidir y actuar por sí mismos.

En este punto, es importante incluir los planteamientos de Henson y Eller (2000), Woolfolk (2006), sobre varios retos que deben ser asumidos en líneas y propuestas de investigación y desarrollo desde el rol del psicólogo en el contexto educativo y entre las cuales de manera resumida encontramos: (1) Reflexiones permanentes en los cambios sociales, políticos y culturales, que produzcan impacto y reestructuración de los sistemas educativos, (2) Los problemas de pobreza, marginación y exclusión que vive la población escolar para plantear la necesidad de la estructuración de estrategias acordes con una educación de calidad para estos contextos, (3) Crear conciencia sobre la importancia continua que adultos responsables acompañen el proceso escolar (4) La transformación de la política educativa, (5) La discriminación racial, cognitiva, desigualdad de género entre otras, debe ser un factor clave para el cambio de la educación que busque la igualdad y la libertad, (6) El mejoramiento del clima escolar, que produzca soluciones a los problemas de convivencia, pandillas y grupos sociales, 
que atentan contra los derechos de los demás, siendo uno de los temas de necesidad para el abordaje, no desde estrategias de discriminación, castigo o expulsión, si no dese la oportunidad de ofrecer currículos y programas que puedan acceder a estudiantes con dificultades de comportamiento. (Erazo,2010), (8) Estudiantes desplazados, la violencia, el conflicto, la falta de empleo, el divorcio, el madres cabeza de familia y su tratamiento dentro del aula y sus formas de manejo en el aula, (9) Los programas de formación en la prevención de malos hábitos como el consumo de drogas, alcohol y cigarrillo y también en sexualidad, que eviten la sexualidad temprana, el VIH - Sida, el embarazo no deseado, entre otras, (10) La comprensión de la cultura cambiante de los educandos, los cuales necesitan una educación acorde con sus necesidades y que puedan prevenir los problemas de suicidio, dificultades de personalidad, desarrollo social y moral (Ardila,1993; Erazo, 2010).

La acción psicosocial

Hasta aquí, ha quedado claro que atender las demandas del entorno educativo es una tarea compleja. Es por esto, que el programa de psicología de la UNAD, con su énfasis social comunitario, pretende dar una mirada amplia a la labor del psicólogo en el contexto educativo, privilegiando la acción psicosocial, más allá de la mera intervención individual o reduccionista.

En este sentido, es preciso comprender aspectos centrales de la acción psicosocial, la cual, acorde con Villa Gómez (2012), permea por lo menos, cuatro niveles: Subjetivo (individual), Interpersonal (familiar, grupal ó comunitario), Sociopolitico (Reconocimiento del individuo 
como ciudadano y como parte de una comunidad y una sociedad, en donde ejerce deberes y derechos) e Histórico cultural (permeado por los procesos educativos y comunicativos que se dan en un espacio y un tiempo particulares).

Por tanto, es importante que el trabajo en comunidades educativas parta del reconocimiento de la intersubjetividad, como lo anota Freire, orientada a la "conciencia de mundo" o la "concienciación" en la medida en que el sujeto es "consciente de sí, del otro y del mundo: Conciencias de un cierto mundo en común” (Freire, 1975); esta conciencia del mundo sólo es posible a partir del diálogo, la palabra, el encuentro con el otro en el lenguaje y su historización, entonces, cuando el sujeto identifica que el mundo está por construirse y que ellos mismos como seres que también lo están y no aceptan el futuro como algo preestablecido es cuando surge la conciencia del mundo, "Tomando conciencia de la situación los hombres se apropian de ella como realidad histórica y por lo tanto capaz de ser transformada por ellos" (Freire, 1975).

El contexto, es entonces, esa realidad del mundo de los sujetos, lo que viven en su cotidianidad, en sus familias, en la escuela, en sus grupos o comunidades; es la realidad de su mundo inmediato en el que "están siendo". Es por ello que los sujetos de la comunidad educativa deben reflexionar sobre la realidad concreta de su territorio a través del conocer, comprender, aplicar, analizar, sintetizar y evaluar las problemáticas o factores de riesgo que están afectando su bienestar biopsicosocial así como el reconocimiento de sus factores protectores y fortalezas, 
a partir del diálogo y la concienciación, las cuales junto con el compromiso, los ayude a la praxis transformadora de carácter político en la educación.

Para una comunidad educativa cada acción psicosocial que se desarrolle debe tener un Sentido y un Significado que se conviertan en fuentes fundamentales del aprendizaje individual y colectivo, ya que lo que se enseña sólo puede ser aprendido en la medida en que convoque a los dos (sentido y significado), en la medida en que represente algo significativo de su realidad concreta, de su experiencia vital, a su conformación como sujetos sociales determinados por una realidad. Aquí, la invitación es revertir el orden de la realidad, la cual no determina al sujeto, sino que es susceptible de ser determinada por él.

La acción psicosocial se comprende como una mirada, una perspectiva y, al mismo tiempo, una forma de enfocar la realidad que posibilita escenarios de actuación que comprenden los fenómenos humanos desde una visión integral y que, por lo tanto, plantean alternativas, soluciones y procesos incluyentes que se despliegan en el tiempo y que producen nuevas subjetividades en marcos de mayor justicia, equidad, desarrollo a escala humana, sana convivencia y paz (Villa, 2012).

El papel del psicólogo en los contextos educativos 
Las vivencias y experiencias obtenidas por el entorno o por el mismo sujeto, son elementos que fundamentan el sentido de vida del ser humano, por ende, es importante que cada aporte que se hace conlleve a la interpretación, el análisis y la comprensión de la realidad, permitiendo avanzar en la transformación de pensamiento y a la vez, romper con paradigmas que coartan la libertad de expresión y señalan al hombre como un ser destructor de su propio entorno y no se reconoce al sujeto como un agente de cambio a partir de posturas críticas y reflexivas que complementa su actuar y promueven ambientes sanos desde lo personal, familiar, social y laboral.

Es por esto que el psicólogo debe trascender los modelos tradicionales, debe colocar su propia impronta que rompa paradigmas estandarizados, que sea capaz de convocar y empoderar a la comunidad de su realidad, que invite a reflexionar sobre ella desde el pensar y repensar a través de la acción psicosocial para transformarla. En este sentido, la acción psicosocial en el contexto educativo aporta a la comprensión y transformación de situaciones problemáticas cada vez más urgentes de comprender e intervenir, siendo así una estrategia de acompañamiento comprometida con el trabajo comunitario, creando sujetos de derecho con el fin de fortalecer su capacidad de autogestión, que orienten a la comunidad a mejorar su bienestar, generar proyectos y rutas de atención integral, que coadyuven en la regulación, potenciación y/o eliminación de aquellos factores psicosociales que de manera directa e indirecta afectan los ambientes escolares y sus poblaciones. 
Igualmente, el psicólogo debe tener claro que en los ambientes educativos existen complejas problemáticas sociales, por consiguiente, debe estar convencido de que su rol será un factor que ayude en la construcción de planes, programas, proyectos y propuestas que hacen parte de la acción psicosocial y se construyen con participación activa de los actores del contexto. De esta manera tanto el psicólogo como los actores implicados podrán articularse y así dar respuesta a los objetivos y metas proyectadas, a fin de promover ambientes escolares saludables que contribuyan en el aprendizaje escolar del sujeto, su formación como persona, el cambio de paradigmas y finalmente el mejoramiento de su calidad de vida de la comunidad educativa, en donde la acción psicosocial se conciba desde y para la comunidad, tal como lo evidencia la educación en sí misma.

Es por eso que la educación debe ser emancipadora, debe configurar las subjetividades e intersubjetividades para que sean reflexivas y deliberativas, debe ayudar a construir a los diferentes actores como "sujetos concretos". El sujeto concreto es aquel que tiene conciencia de lo ocurrido, tiene conciencia histórica ya que ello permite comprender la realidad porque ello compromete la creación de pensamiento.

Procesos y actividades en la acción psicosocial en el contexto educativo

El psicólogo en el contexto educativo tiene la responsabilidad de acompañar y apoyar los procesos educativos, las interacciones y el fortalecimiento del sentido de comunidad, así, se pueden identificar diferentes funciones del psicólogo en el contexto educativo: 
1. Articular elementos y procesos educativos para favorecer el aprendizaje significativo.

2. Participar en la construcción del PEI (Proyecto Educativo Institucional) en las instituciones educativas para contribuir a la regulación del servicio educativo que corresponda a las necesidades del desarrollo humano y a la urgencia de los problemas sociales contemporáneos, especialmente en la construcción de los proyectos que son transversales en la comunidad educativa especialmente donde el psicólogo debe ser un orientador de los procesos, teniendo en cuenta las necesidades propias de cada institución educativa.

3. Contribuir al mejoramiento del proceso educativo, que incluye lo pedagógico y lo curricular, lo que implica apoyo en la orientación escolar y acciones de asesoría a la comunidad educativa sobre las problemáticas escolares que emergen en esa comunidad.

4. Fomentar la investigación en el contexto educativo para fortalecer sus procesos. En este aspecto, podemos mencionar aquí: “Artículo 1º. Definición. La Psicología es una ciencia sustentada en la investigación y una profesión que estudia los procesos de desarrollo cognoscitivo, emocional y social del ser humano, desde la perspectiva del paradigma de la complejidad, con la finalidad de propiciar el desarrollo del talento y las competencias humanas en los diferentes dominios y contextos sociales tales como: La educación, la salud, el trabajo, la justicia, la protección ambiental, el bienestar y la calidad de la vida. Con base en la investigación científica fundamenta sus conocimientos y los aplica en forma válida, ética y responsable en favor de los individuos, los grupos y las organizaciones, en los distintos ámbitos de la vida individual y social, al aporte de conocimientos, técnicas y procedimientos para crear 


Apoyo al trabajo pedagógico
Participa en el diseño de programas educativos en los diferentes niveles, desde la educación infantil hasta la educación profesional, promoviendo estrategias pedagógicas centradas en el aprendizaje.

Desarrolla proyectos educativos institucionales para implementar estrategias innovadoras en la búsqueda constante de la calidad educativa.

Participa en procesos formativos del trabajo docente mediante el desarrollo de estrategias didácticas que fomenten el aprendizaje significativo, contextualizado y aplicado a situaciones reales.

Orientación

Fomenta y favorece la implementación de la orientación psicoeducativa educativa en los centros de enseñanza para guiar a padres, profesores, y de manera especial a los estudiantes, para que éstos se conozcan a sí mismos y sepan cuáles son sus posibilidades en cuanto al aprendizaje. 
Acompañamient

o a los procesos de

Inclusión educativa
Se involucra con el personal docente, directivo y padres de familia para la integración de estudiantes con necesidades educativas especiales mediante la sugerencia de estrategias para el desarrollo de sus potencialidades.

\begin{tabular}{l} 
Desarrollo $\begin{array}{c}\text { Participa en procesos formativos no escolarizados en } \\
\text { personal }\end{array}$ \\
programas de prevención y correctivos para que los sujetos logren \\
su desarrollo personal e integración a la sociedad. \\
Prólogos) relacionados con la formación académica a todos los \\
agentes educativos, incluyendo a los padres de familia. \\
\hline Investigación \\
profesionales y laborales para acrecentar el desarrollo del \\
conocimiento, propio de la especialidad así como registrar los \\
ámbitos de intervención profesional.
\end{tabular}

Fuente: Adaptado de Hernández, 2008.

En este orden de ideas, la acción psicosocial en el contexto educativo se puede enmarcar desde varios frentes epistemológicos que generen diversos modelos de acción entendida esta última como, “...una acción centrada en la promoción de prácticas relacionales basadas en el reconocimiento y la valoración del ser humano en ejercicio de construcción. Todos estos 
elementos interactúan sistémicamente y son fundamentales a la hora de comprender y abordar lo psicosocial” (González \& Villa, 2012).

Métodos y técnicas para la acción psicosocial en el contexto educativo

Al llegar a una institución educativa por lo general se observan problemáticas escolares, e inmediatamente se propone o diseñan proyectos o programas para intervenir sin tener en cuenta el contexto particular, el pensar y el sentir de cada uno de los miembros de la institución, sin buscar la participación activa de los actores desde el diálogo de saberes para luego, al evaluar las estrategias, encontrar que el impacto fue parcial o nulo.

Partiendo de lo anterior se hace necesario que el psicólogo utilice una metodología participativa y contextualizada, que permita construir procesos que conlleven a cambios significativos en la comunidad educativa y para esto debe enfatizar en:

1. Metodología cualitativa, esto es, procedimientos para conocer la realidad, para realizar aproximaciones a esa realidad compleja, sin que se aspire a una verdad como adecuación, ni a la construcción de leyes y teorías universales explicativas de los fenómenos, sino a interpretaciones densas de los mismos (Geertz, 1989). En términos de acción se concibe que estrategias de acompañamiento y acción relacionales (familiares, colectivas/comunitarias y 
públicas) pueden tener una incidencia más profunda sobre la construcción y la transformación de la subjetividad de quienes participan en las mismas.

2. Reconocimiento participativo de las realidades de la comunidad educativa a través de un diagnóstico contextual.

3. Identificación de las capacidades instaladas y requeridas para gestar las transformaciones. En este aspecto, existen grandes desafíos para el psicólogo educativo, pues apoyarse en los fundamentos teóricos de la psicología y de la educación, que le den soporte a su actuar bajo una actualización permanente, que le permitan su autogestión y generación de cambios significativos que contribuyan a un desarrollo y progreso comunitario, desde el análisis de lo existente en el contexto mismo.

4. Reconocimiento de las posibilidades de la comunidad para apoyar y acompañar procesos de movilización.

5. Después de conocer esa realidad, el psicólogo debe apoyarse en métodos que se ajusten a las características sociales, históricas y culturales de los contextos y a las características de desarrollo de los diferentes actores, que permitan indagar, conocer y acercarse a los entornos educativos donde se recrean diferentes factores psicosociales y educativos que influyen directa e indirectamente en las actitudes de los sujetos de manera individual y colectiva, por 
ende, el enfoque, el diseño, las herramientas, estrategias o técnicas a utilizar deben ser acordes con la situación presentada que permitan participar de manera activa a todos los miembros de la comunidad en el ejercicio de la concienciación y de reflexión, para diseñar conjuntamente planes, programas, proyectos y redes de apoyo encaminados a generar empoderamiento, transformación y bienestar comunitario.

Esta ruta metodológica se debe comprender la realidad desde las subjetividades e intersubjetividades del contexto educativo, desde una visión histórica, sociocultural, económica y política que identifique las problemáticas escolares que afectan al individuo, a grupos y a la comunidad en general para construir procesos acción psicosocial, desde una perspectiva holística, permitiendo así el mejoramiento de la calidad de vida de los miembros de la comunidad escolar.

Sin embargo, no se puede descartar que en algunos momentos el psicólogo realice acciones dirigidas a situaciones particulares que ameriten una intervención inmediata pero que no atenten contra los derechos de los miembros de la institución educativa de acuerdo con las normas.

Resumiendo lo anterior, el psicólogo debe empoderar a la comunidad educativa para que sea autogestora de su propio cambio y esas construcciones colectivas deben desencadenarse en las potencialidades que tiene el sujeto, basadas en la capacidad de observar, preguntar, reflexionar y tomar conciencia de las realidades y a partir de allí, construir, de construir o 



\section{Referencias Bibliográficas}

Villa Gómez, J.; (2012). La acción y el enfoque psicosocial de la intervención en contextos sociales: ¿podemos pasar de la moda a la precisión teórica, epistemológica y metodológica? EL ÁGORA USB, Julio-diciembre.

UNAD. (2013). Documento condiciones de calidad del programa de psicología con fines de Renovación de registro calificado elaborado de conformidad con el decreto 1295.

Freire, Paulo. (1978). La educación como práctica de la libertad. Siglo XX editores. México.

Henson, K. T., \& Eller, B. F. (2000). Como ayuda la psicología educativa a los profesores y estudiantes. En: Psicología Educativa para la Enseñanza Eficaz. México: Thompson.

Maturana, H. (1998). El sentido de lo humano (1ra Ed.). Bogotá: Dolmen Editores.

Hernández Madrigal, P. (2008) Los campos de acción del psicólogo educativo, en Psicología Científica [Disponible en] http://www.psicologiacientifica.com/bv/psicologiapdf-321los-campos-de-accion-del-psicologo-educativo.pdf. (Recuperado: 11 de octubre de 2010). 


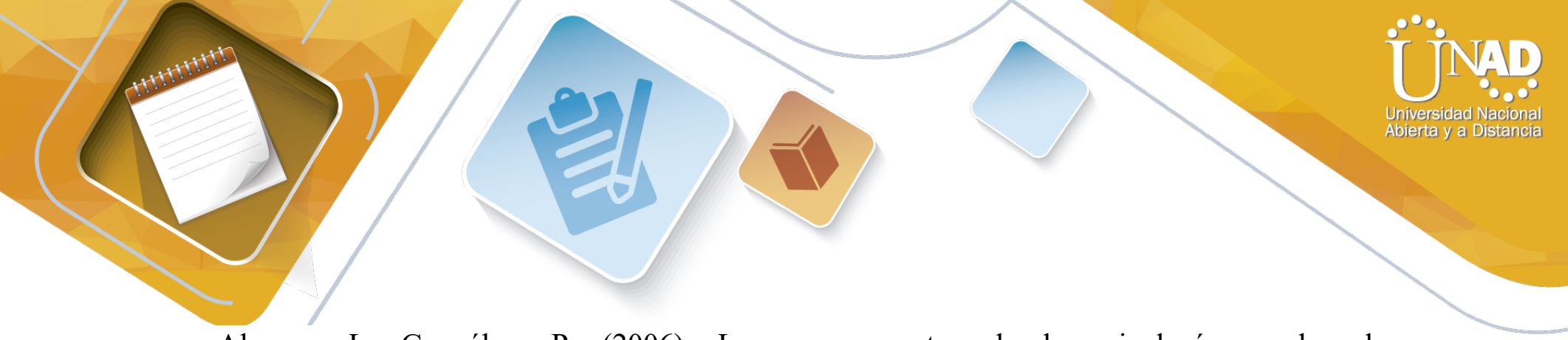

Alvarez, L; González, P. (2006). Los nuevos retos de la psicología escolar: la orientación. Papeles del Psicólogo, septiembre-diciembre, 185-191. Recuperado de http://www.redalyc.org/articulo.oa?id=77827308. 